\title{
Efficacy of pentoxifylline in Peyronie's disease: Clinical case of a young man
}

\author{
Lucio Dell'Atti, Gianni Ughi \\ Urology Unit, Arcispedale "S. Anna", University of Ferrara, Italy.
}

\begin{abstract}
Summary Peyronie's disease (PD) is a localized connective tissue disorder of the tunica albuginea of the penis and its surrounding tissue which results in a painful erection, penile curvature and erectile dysfunction. The great number and variety of purposed treatments for PD is in proportion to the difficulty of its management.In fact no medical treatment is currently available to cure patients with $P D$.

Pentoxifylline (PTX) is a non specific phosphodiesterase inhibitor with anti-inflammatory properties that has been used to treat claudication. PTX has also been used to decrease inflammation and fibrosis in kidney transplants, open heart surgery, dermatological conditions and after radiation injury. With respect to penile diseases, clinical studies have suggested that PTX decreases calcification in new-onset PD. These traits make PTX an interesting potential option for PD therapy.
\end{abstract}

KEY WORDS: Peyronie's Disease; Pentoxifylline; Ultrasound; Phosphodiesterase inhibitor.

Submitted 5 September 2013; Accepted 30 June 2014

\section{INTRODUCTION}

Peyronie's disease (PD) is a relatively common disorder in men (3-9\%) and a frequent cause of sexual distress (1). PD (or Induratio Penis Plastica) is characterized by the formation of a fibrous plaque within the tunica albuginea of the penile corpora cavernosa. Most patients present with concerns about a penile lump, curvature, painful erections or erectile dysfunction. A short list of differential diagnoses, such as congenital chordee, dorsal vein thrombosis, infiltrative cancer or a sexually transmitted disease need to be excluded. Oral pharmacotherapy should be considered as a treatment option for acute and earlier chronic phases. Many oral medicines, including vitamin E, potassium para-aminobenzoate, tamoxifen, colchicines, propionyl-Lcarnitine, have been used, but there is no satisfactory oral medical treatment available (2).

Pentoxifylline (PTX) has anti-inflammatory and antifibrogenic properties, with inhibitory effects on the basic mechanisms of fibrogenesis, cell proliferation and extracellular matrix synthesis (3). These mechanisms of actions might reverse the fibrotic process in PD.

\section{Case report}

A 35 years old Caucasian male had for about 5 years difficulties in penetration during sexual act due to a lack of rigidity and tumescence in the terminal part of the penis. However the patient during erection did not report the presence of curving of the penis. This disorder was not associated with erectile dysfunction, ejaculatory dysfunction or low libido. There is no personal or family history of fibrotic disorders. He does not smoke and has an occasional alcoholic beverage, moreover his general health was excellent and he doesn't make use of medicines or drugs.

Physical examination revealed nothing abnormal in his testicles, epididymis or scrotum. However, a large fibrotic lesion was palpable in correspondence of the dorsal portion of the penis approximately below the root to the glans.

Laboratory evaluation was normal including complete blood count, routine biochemistry analyses, kidney and liver function tests, sex hormones, prolactin and serum lipid measurement. Penile B-mode sonography revealed a calcified Peyronie plaque that involved about $1 \mathrm{~cm}$ in length below the glans the entire tunica albuginea of both corpora cavernosa.

This plaque created a deep notch on the medial profile that in addition to affect both the corpora cavernosa on one side penetrated ventrally causing dislocation and compression of the cavernous arteries, while on the other side back penetrated leading to removal of albuginea from Buck's fascia (Figure 1).

To evaluate the penile vasculature, an intracavernosal injection with $20 \mu \mathrm{g}$ of prostaglandin El was administered and color and duplex Doppler sonography were performed during tumescence.

The study revealed a peak systolic flow velocity (PSV) of $60 \mathrm{~cm} / \mathrm{s}$ (Figure 2), an end-diastolic flow velocity (EDV) of $4 \mathrm{~cm} / \mathrm{s}$ with resistivity index (RI) 0,93 in the cavernosal arteries below the plaque, while in the cavernosal arteries above the plaque PSV was $20 \mathrm{~cm} / \mathrm{s}$ (Figure 3), EDV 5 $\mathrm{cm} / \mathrm{s}$ and RI 0.73 .

The patient was given sildenafil citrate at 50 and $100 \mathrm{mg}$ orally for addressing erectile dysfunction without any benefit in terms of tumescence of the terminal part of the penis. The patient was prescribed PTX $400 \mathrm{mg}$ three times a day for 6 months. Upon re-evaluation 6 months 
later, the patient reported improvement of tumescence of the glans with the possibility to have penetration during sexual intercourse in association of occasional use of 5-

Figure 1.

Penile B-mode sonography revealed a calcified Peyronie plaque.

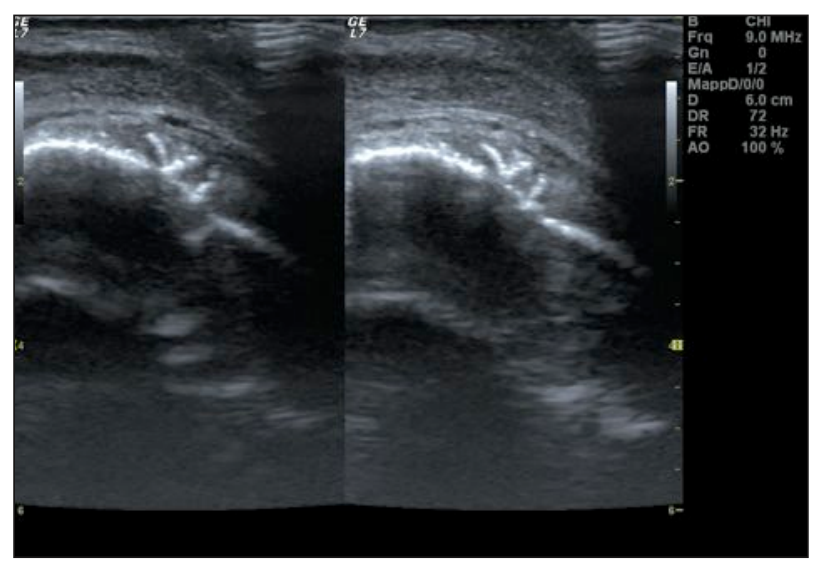

Figure 2.

Penile color-Doppler sonography revealed a PSV of $60 \mathrm{~cm} / \mathrm{s}$ in the cavernosal arteries below the plaque.

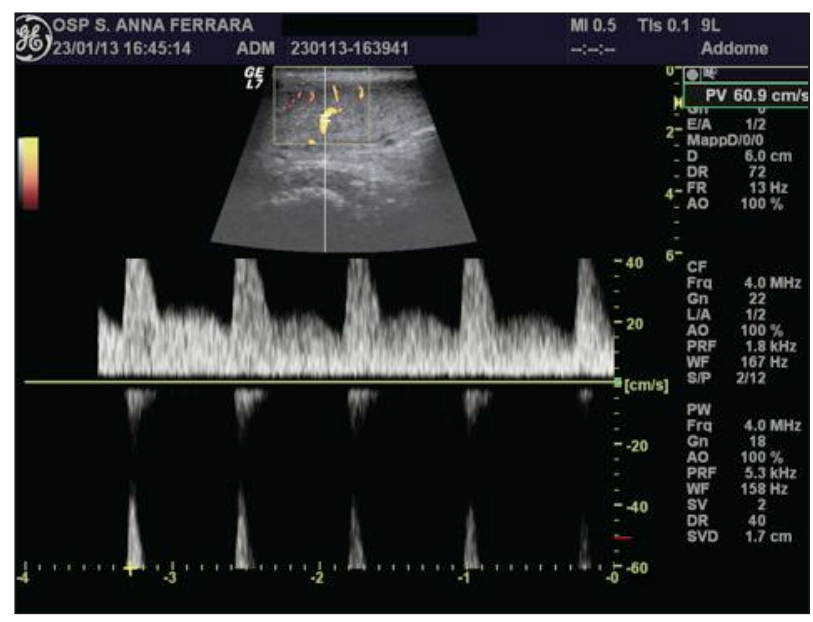

\section{Figure 3.}

Penile color-Doppler sonography revealed a PSV of $20 \mathrm{~cm} / \mathrm{s}$ in the cavernosal arteries above the plaque.

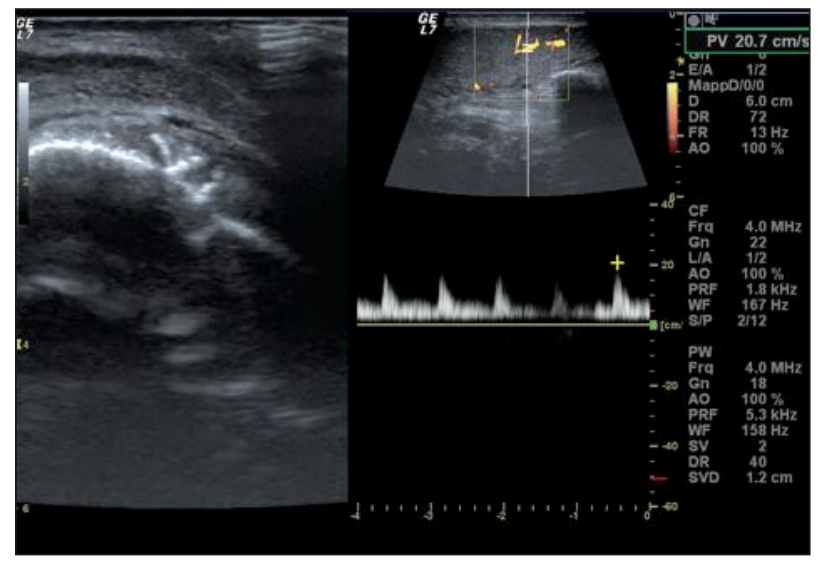

phosphodiesterase inhibitors. Pentoxifylline use was continued, and, upon reassessment one years later, improved erectile function was reported without the use of erectogenic agents.

\section{Conclusion}

The management of patients with PD would be improved by the development of a treatment strategy that can reverse the abnormal fibrotic reaction of the tunica albuginea. Although the case reported certainly presents as an atypical form of PD, it was improved by PTX administration that increased tissue perfusion and PSV and showed anti-inflammatory and antifibrogenic properties, with inhibitory effects on the basic mechanisms of fibrogenesis, e.g. cell proliferation and extracellular matrix synthesis.

We believe that more studies are required to determine the optimal doses and treatment duration.

\section{REFERENCES}

1. Schwarzer U, Sommer F, Klotz T, et al. The prevalence of Peyronie's disease: results of a large survey. BJU Int. 2001; 88:727-30.

2. Abern MR, Larsen S, Levine L. Combination of Penile traction, Intralesional Verapamil and oral therapies for Peyronie's Disease, J Sex Med. 2012; 9:288-295.

3. Windmeier C, Gressner AM. Pharmacological aspects of pentoxifylline with emphasis on its inhibitory actions on hepatic fibrogenesis. Gen Pharmacol. 1997; 29:181-96.

Discussion and full list of References are posted in Supplementary materials on www.aiua.it

Correspondence

Lucio Dell'Atti, MD, PhD (Corresponding Author) dellatti@hotmail.com

Gianni Ughi MD gianniughi@ospfe.it

Urology Unit, Arcispedale "S. Anna"

Via A. Moro 8 - 44124 Cona, Ferrara, Italy 